\title{
On the Use of Absorbing Metasurfaces in Microwave Imaging
}

\author{
Ziqi Liu, Nozhan Bayat, and Puyan Mojabi
}

\begin{abstract}
Microwave imaging (MWI) systems are usually enclosed within casings, e.g., in order to contain the utilized coupling liquid or to help mount the antenna system. On the other hand, inverse scattering algorithms, which are used to process the measured microwave scattering data, often assume that the background medium of the imaging system extends to infinity (i.e., unbounded background medium assumption). Thus, they do not consider the reflections occurring at the system enclosure. For such algorithms to yield successful images, these reflections need to be minimized, e.g., via the use of a lossy coupling liquid. As an alternative to a lossy background medium which also reduces the desired signal level, this paper investigates the use of metallic-backed absorbing metasurfaces as the MWI system enclosure in order to (i) reduce these reflections, and also (ii) to shield the MWI system from external interference. Using simulated data, we then show that standard inverse scattering algorithms, employing the free-space assumption, can successfully process the data collected under the metasurface enclosure and yield acceptable permittivity images. The advantages and disadvantages of absorbing metasurface enclosure, along with the limitations of this study, will also be discussed. Finally, an absorbing metasurface is fabricated and its reflectivity is experimentally evaluated.
\end{abstract}

Index Terms-Microwave imaging (MWI), inverse scattering, absorbing metasurfaces.

\section{INTRODUCTION}

$\mathbf{M}$ ICROWAVE imaging (MWI) is a non-invasive and nonionizing imaging modality that creates qualitative or quantitative images of the relative complex permittivity profile of an object of interest (OI) [1]-[5]. The inverse scattering approach to MWI, which is the focus of this paper, is usually concerned with reconstructing a quantitative image of the OI's relative complex permittivity profile. In this approach, the OI is often irradiated from different angles, and the resulting scattered fields are collected at different locations around the OI. These external scattered field data are then processed by an inverse scattering algorithm to reconstruct a quantitative image of the OI's relative complex permittivity profile. To this end, the inverse scattering approach employs a nonlinear optimization scheme to iteratively reconstruct a quantitative relative complex permittivity profile by minimizing the discrepancy between the simulated scattering data from a predicted permittivity image and the measured scattered data from the true

This paragraph of the first footnote will contain the date on which you submitted your paper for review. The financial support of the Natural Sciences and Engineering Research Council (NSERC) of Canada, University of Manitoba's UMGF and GETS Program, as well as the Canada Research Chair (CRC) Program is acknowledged.

The authors are with the Department of Electrical and Computer Engineering, University of Manitoba, Winnipeg, MB, Canada (e-mail: liuz3421@myumanitoba.ca).
OI. Two common inverse scattering algorithms, which are used in this paper, are the Gauss-Newton inversion (GNI) [6], [7] and contrast source inversion (CSI) algorithms [8].

Based on the above optimization approach to MWI, it can be understood that the success of the inverse scattering algorithms relies on the following necessary condition: if the true model of the $\mathrm{OI}$ is given to the computational electromagnetic solver used in the inverse scattering algorithm, the resulting simulated scattered data need to be sufficiently close to the measured data. However, since the true OI is the actual unknown of the problem, this can never be checked in practice. Instead, the above requirement is often checked in the absence of the OI, i.e., comparing the simulated incident field with the measured incident field. Alternatively, a known target can be employed to compare its corresponding simulated and measured scattered field data. (This comparison of the simulated and measured data is done as part of the data calibration step in microwave imaging [9].)

One of the sources of discrepancy between the simulated and measured data is the reflections from the microwave imaging system enclosure (casing). For example, consider the microwave biomedical imaging experiments considered in [10] in which water was used as the coupling liquid to help with coupling incident microwaves into biological tissues. The use of this coupling liquid fluid reduces the overall impedance mismatch between the OI and the background medium. However, this comes with the following challenge. Assume that the enclosure of the MWI system is an electrically thin plexiglass. Therefore, at the enclosure of the MWI system, we now have a significant impedance mismatch between the interface of water (inside the enclosure) and air (outside the enclosure). Alternatively, if the enclosure is a perfect electric conductor (PEC), we will have $100 \%$ reflection.

One common method to handle the above challenge is to make the coupling liquid (also known as matching fluid) lossy to reduce the level of the reflected signals [10]-[13] 1 By adjusting the coupling liquid loss level as well as the separation between the antennas and the enclosure, the power of these reflected waves can be made negligible once arrived at the receiving antennas. This enables the microwave imaging algorithm to assume that the coupling liquid extends to infinity (i.e., Sommerfeld radiation condition). Therefore, the green's function of an unbounded homogeneous medium can be used in the implementation. Due to the convolutional form of such

\footnotetext{
${ }^{1}$ Other methods such as taking into account these reflections in the imaging algorithm or calibration techniques to remove the undesired fields also exist [14], [15], which is not within the scope of this paper.
} 
green's function ${ }^{2}$ efficient computational solvers, e.g., the method of moments with the conjugate gradient algorithm accelerated by the Fast Fourier transform (CG-FFT MoM) can then be used in the MWI algorithm.

However, a lossy coupling liquid not only reduces the level of the reflections from the enclosure but also reduces the level of the desired signal. To understand this better let us consider [12] in which water with various additions of table salt was used to create coupling liquids with various loss levels. (The greater the addition of table salt, the more lossy the coupling liquid.) For small addition of table salt, the loss level was not sufficient to reduce the reflections to an acceptable level. On the other hand, beyond a certain level of salt addition, the loss level was too high to the extent that the desired signal was not usable (i.e., a weak signal-to-noise ratio). Therefore, an appropriate loss level between these two limits was chosen to perform imaging. (Typically, in MWI around the frequency of $1 \mathrm{GHz}$, a loss value corresponding to a conductivity of $\sigma \approx 1 \frac{\mathrm{S}}{\mathrm{m}}$ has been used.) As another example, in [13], the conductivity of $\sigma=1.13 \frac{\mathrm{S}}{\mathrm{m}}$ has been used for the coupling liquid for microwave breast imaging. These typical values for the conductivity level correspond to the following imaginary part values for the relative complex permittivity of the coupling liquid: between -15 to -20 assuming $\exp (j \omega t)$ time dependency.

In this paper, the purpose is to investigate the use of absorbing metasurfaces as the enclosure of MWI systems in an attempt to relax the necessity of having a high-loss background medium for the imaging system. Electromagnetic metasurfaces [16]-[18] are thin structures consisting of subwavelength elements with various applications such as tailoring radiation patterns [19], refraction [20], polarization control [21], absorption [22], etc. Once illuminated, electric and magnetic equivalent currents are induced on these electromagnetic metasurfaces that can then tailor the incident electromagnetic wave in various ways. For example, in [23], currents are induced on three layers of metallic dogbone traces on two bonded Rogers RO3010 substrates to refract a normally incident plane wave by $70^{\circ}$. (The thickness of this metasurface is about $2.5 \mathrm{~mm}$ at the frequency of $9.53 \mathrm{GHz}$, i.e., less than $\lambda_{0} / 12$ where $\lambda_{0}$ is the wavelength in free space.) The category of metasurfaces considered herein is the absorbing metasurfaces which are often designed so as to have a small reflection coefficient for a normally incident plane wave [22], [24]-[26]. For such absorbing metasurfaces, once the incident plane wave deviates from the normal incidence, the reflection coefficient worsens as compared to the normal incidence.

The potential advantages of these absorbing metasurfaces for microwave imaging system enclosure are the followings.

- They are electrically thin and light weight (e.g., in the order of one or two dielectric substrates). Therefore, they help with the portability of the MWI system;

- They are often metallic backed. Thus, they can shield the MWI system from undesired external interference and cable effects;

\footnotetext{
${ }^{2}$ That is, the green's function will be $g\left(\mathbf{r}, \mathbf{r}^{\prime}\right)=g\left(\mathbf{r}-\mathbf{r}^{\prime}\right)$ where $\mathbf{r}$ and $\mathbf{r}^{\prime}$ are the observation and source locations respectively.
}

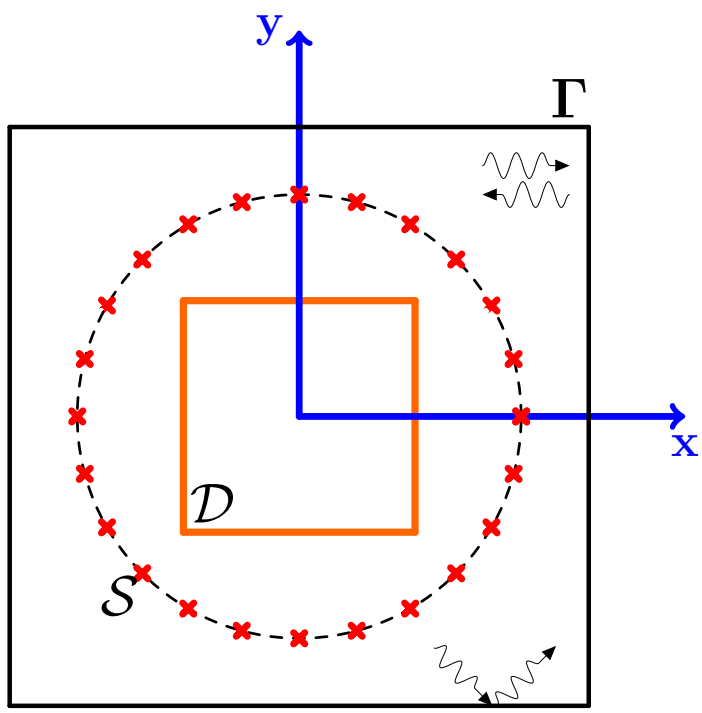

Fig. 1. The schematic of a microwave imaging system consisting of three main geometrical domains: (i) the imaging domain $\mathcal{D}$, (ii) the measurement domain $\mathcal{S}$ (the red crosses show the locations of antennas on $\mathcal{S}$ ), and (iii) the enclosure of the system $\Gamma$. We might have some reflections from $\Gamma$ back to the imaging environment.

- Since the absorption occurs at the metasurface, the loss of the coupling liquid (background medium) can be reduced. This can improve the signal to noise ratio (SNR) of the measured data. (An improved SNR enhances the achievable image resolution and accuracy.)

- When the background medium is air (e.g., see [27]) the absorbing metasurface enclosure still shields the imaging domain from external noise (due to its PEC backed structure), while not creating significant reflections back to the imaging domain.

On the other hand, a disadvantage of an absorbing metasurface enclosure is its limited bandwidth, which inhibits its use in wideband MWI systems. However, it does not create any major issue for narrow-band MWI systems. A second challenge with absorbing metasurfaces is the angular dependency of their absorption. As will be seen, despite their angular dependency, they can still be helpful in reducing the reflections. In what follows, we first go over MWI, and our problem statement as well as some aspects of metasurfaces including their generalized sheet transition conditions in terms of surface susceptibilities. We will then consider three unit cell designs for absorbing metasurfaces which will later be used as our MWI enclosures. Based on these unit cells, we present some synthetic imaging results using these absorbing metasurface enclosures with the data collected in Ansys HFSS to demonstrate their performance. Finally, we show a fabricated metasurface and its associated experimental test. (This paper is the extended form of our conference paper [28].)

\section{Microwave Imaging (MWI)}

Consider Figure 1 which shows a schematic of an MWI system. The imaging domain, in which the OI resides, is denoted by $\mathcal{D}$. The measurement domain located outside the imaging 
domain is denoted by $\mathcal{S}$, and represents the location of the transmit/receive antennas. Finally, the enclosure of the MWI system is denoted by $\Gamma$. This enclosure, which can for example be plexiglass [27] or metal [10], causes some reflections back to the imaging environment. Under tomographic MWI (i.e., 2D transverse magnetic $\mathrm{TM}_{z}$ propagation), we assume that the imaging domain lies in the $x y$ plane, and the imaging is performed with the $z$ component of the electric field (and subsequently with the $x$ and $y$ components of the magnetic field). Thus, the main governing equation of the MWI process can be expressed as

$$
\nabla^{2} E_{z}^{\text {scat }}+k_{b}^{2} E_{z}^{\text {scat }}=-k_{b}^{2} w, \text { (in the imaging system) }
$$

$E_{z}^{\text {scat }}$ subject to the boundary condition on $\Gamma$

where $\nabla^{2}$ denotes the Laplacian operator, $k_{b}$ is the wavenumber in the background medium, and $w$ denotes the unknown contrast sources [8] in the OI. Finally, $\boldsymbol{E}^{\text {scat }}=E_{z}^{\text {scat }} \hat{z}$ denotes the scattered field $\hat{\jmath}^{3}$ which has only the $\hat{z}$ component under our tomographic assumption. Assuming that the reflections by the enclosure $\Gamma$ is small when arrived at the antennas, we can replace the boundary condition in (1) by Sommerfeld radiation condition, and arrive at the so-called data equation [8]

$$
E_{z}^{\text {scat }}(\boldsymbol{r} \in \mathcal{S})=k_{b}^{2} \int_{\mathcal{D}} g\left(\boldsymbol{r}, \boldsymbol{r}^{\prime}\right) O\left(\boldsymbol{r}^{\prime}\right) E_{z}\left(\boldsymbol{r}^{\prime}\right) d \boldsymbol{r}^{\prime}
$$

where $g$ denotes the green's function of the background medium. Due to the assumption that the background medium extends to infinity, we then have

$$
g\left(\boldsymbol{r}, \boldsymbol{r}^{\prime}\right)=g\left(\boldsymbol{r}-\boldsymbol{r}^{\prime}\right)=\frac{1}{4 j} H_{0}^{2}\left(k_{b}\left|\boldsymbol{r}-\boldsymbol{r}^{\prime}\right|\right),
$$

where $H_{0}^{2}$ is the zeroth order Hankel function of the second kind. ( $\boldsymbol{r}$ and $\boldsymbol{r}^{\prime}$ denote the position vectors.) In addition, $O(\boldsymbol{r})$ denotes the main unknown of the problem: the relative complex permittivity contrast function (object function) defined as

$$
O(\boldsymbol{r}) \triangleq \frac{\epsilon(\boldsymbol{r})-\epsilon_{b}}{\epsilon_{b}},
$$

where $\epsilon_{b}$ is the relative permittivity of the background medium and $\epsilon$ is the unknown relative complex permittivity of the object being imaged. Finally, $\boldsymbol{E}=E_{z} \hat{z}$ denotes the induced total field in the imaging domain which is also unknown and is nonlinearly related to $O$ through the so-called domain equation (also known as the state equation) [8]. Due to this nonlinearity, the contrast is updated in an iterative fashion. Two types of algorithms are common in the literature to iteratively reconstruct the relative complex permittivity contrast, namely, Gauss-Newton inversion (GNI) and contrast source inversion (CSI) methods ${ }^{4}$ These methods often use regularization techniques to stabilize the inversion process (i.e., treating the illposedness of the problem) and to enhance image quality. A

\footnotetext{
${ }^{3}$ The scattered field is defined as the subtraction of the incident field (field in the absence of the OI) from the total field (field in the presence of the OI); i.e., $\boldsymbol{E}^{\text {scat }} \triangleq \boldsymbol{E}-\boldsymbol{E}^{\text {inc }}$.

${ }^{4}$ In both algorithms, the contrast at the $n$th iteration is updated as $O_{n+1}=$ $O_{n}+\beta_{n} u_{n}$ where $O_{n}$ is the known contrast at the $n$th iteration and $\beta_{n}$ is the step length. The vector $u_{n}$ is the conjugate gradient direction at the $n$th iteration of the CSI algorithm, and Newton correction at the $n$th iteration of the GNI algorithm.
}

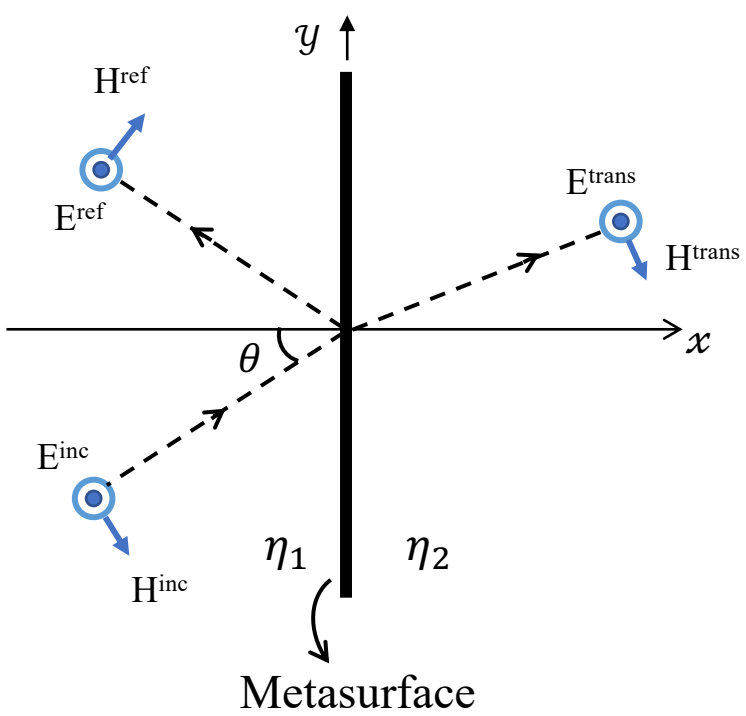

Fig. 2. A metasurface is located on the $y$ axis. Thus, the input and output sides of the metasurface are $x=0^{-}$and $x=0^{+}$respectively. A TM $z$ incident wave illuminates the metasurface which results in reflected and transmitted waves. Also, $\eta_{1}$ and $\eta_{2}$ denote the wave impedance in the two media.

common regularization technique is the weighted $L_{2}$ norm total variation multiplicative regularizer which provides both smoothing and edge-preserving operations. Under this regularization, the above two algorithms are referred to as MR-GNI and MR-CSI algorithms [6]-[8], [10], [29]. As will be seen in Section VII, we use the MR-GNI and MR-CSI algorithms to generate images from microwave scattered data. In all our reconstructions, these algorithms have started from a trivial initial guess which is $O(\boldsymbol{r})=0$ for the MR-GNI algorithm, and the back-propagation solution for the MR-CSI algorithm.

\section{Problem Statement}

The main purpose of this paper is to investigate the use of absorbing metasurfaces for the MWI enclosure $\Gamma$ such that

- we can assume that the background medium extends to infinity, which is equivalent to assuming small reflections from $\Gamma$, thus, enabling the use of (3) as the green's function in our MWI algorithms;

- the imaging system is shielded from external interference, i.e., utilizing a metallic-backed absorbing metasurface.

\section{Metasurface Theory}

Metasurfaces provides a boundary condition for the wave propagation which is described by the generalized sheet transition conditions (GSTCs) [30]-[32]. Assuming that the metasurface is along the $y$ axis as shown in Figure 2, the 
GSTCs relate the tangential fields ( $\hat{y}$ and $\hat{z}$ components) on both sides of the metasurface as 5

$$
\begin{aligned}
{\left[\begin{array}{c}
\Delta H_{y} \\
-\Delta H_{z}
\end{array}\right]=} & j \omega \epsilon_{0}\left[\begin{array}{cc}
\chi_{e e}^{z y} & \chi_{e e}^{z z} \\
\chi_{e e}^{y y} & \chi_{e e}^{y z}
\end{array}\right]\left[\begin{array}{c}
E_{y, \mathrm{av}} \\
E_{z, \mathrm{av}}
\end{array}\right]+ \\
& j \omega \sqrt{\mu_{0} \epsilon_{0}}\left[\begin{array}{cc}
\chi_{e m}^{z y} & \chi_{e m}^{z z} \\
\chi_{e m}^{y y} & \chi_{e m}^{y z}
\end{array}\right]\left[\begin{array}{c}
H_{y, \mathrm{av}} \\
H_{z, \mathrm{av}}
\end{array}\right], \\
{\left[\begin{array}{c}
-\Delta E_{y} \\
\Delta E_{z}
\end{array}\right]=} & j \omega \mu_{0}\left[\begin{array}{cc}
\chi_{m m}^{z y} & \chi_{m m}^{z z} \\
\chi_{m m}^{y y} & \chi_{m m}^{y z}
\end{array}\right]\left[\begin{array}{c}
H_{y, \mathrm{av}} \\
H_{z, \mathrm{av}}
\end{array}\right]+ \\
& j \omega \sqrt{\mu_{0} \epsilon_{0}}\left[\begin{array}{cc}
\chi_{m e}^{z y} & \chi_{m e}^{z z} \\
\chi_{m e}^{y y} & \chi_{m e}^{y z}
\end{array}\right]\left[\begin{array}{c}
E_{y, \mathrm{av}} \\
E_{z, \mathrm{av}}
\end{array}\right],
\end{aligned}
$$

where $\omega, \epsilon_{0}$, and $\mu_{0}$ denote the angular frequency, permittivity and permeability of free space respectively. In the above GSTCs, the position vector $\boldsymbol{r}$ has been dropped for brevity. Assuming a given location on the metasurface, say $\boldsymbol{r}$, $\Delta u=\Delta u(\boldsymbol{r})$ (where $u \in\left\{E_{y}, E_{z}, H_{y}, H_{z}\right\}$ ) represents the difference between the tangential fields on the two sides of the metasurface at $\boldsymbol{r}$; i.e., $\Delta u=u^{+}-u^{-}$where $u^{+}$is the tangential field on the output side (i.e., on $x=0^{+}$) and $u^{-}$ is the tangential field on the input side (i.e., on $x=0^{-}$). On the other hand, $u_{\mathrm{av}}=u_{\mathrm{av}}(\boldsymbol{r})$ is the algebraic average of the tangential fields on both sides; i.e., $u_{\mathrm{av}}=\left(u^{+}+u^{-}\right) / 2$. Then, according to the GSTCs, these tangential fields are related through the surface susceptibilities at location $r$ denoted by $\chi$ where the position vector has been dropped for brevity. These surface susceptibilities represent how an electric $(e)$ or magnetic $(m)$ field excitation (second subscript) in a given direction (second superscript) results in an electric $(e)$ or magnetic $(m)$ polarization response (first subscript) in a given direction (first superscript). For example, consider $\chi_{m e}^{y z}$ : it represents how an electric field excitation in the $z$ direction (i.e., second subscript and superscript) creates a magnetic polarization response in the $y$ direction (first subscript and superscript).

Since we are considering tomographic imaging in the $x y$ plane, we limit ourselves to the two-dimensional (2D) transverse magnetic $\left(\mathrm{TM}_{z}\right)$ propagation as shown in Figure 2 Thus, we only have three field components, namely $E_{z}, H_{y}$, and $H_{x}$. Since $H_{x}$ is perpendicular to the metasurface, it is absent in the GSTCs. This simplifies (5) and (6) to

$$
\begin{aligned}
& \Delta H_{y}=j \omega \varepsilon_{0} \chi_{e e}^{z z} E_{z, a v}+j \omega \sqrt{\mu_{0} \varepsilon_{0}} \chi_{e m}^{z y} H_{y, a v}, \\
& \Delta E_{z}=j \omega \mu_{0} \chi_{m m}^{y y} H_{y, a v}+j \omega \sqrt{\mu_{0} \varepsilon_{0}} \chi_{m e}^{y z} E_{z, a v} .
\end{aligned}
$$

Therefore, the metasurface design problem can be stated as follows: given a set of (desired) tangential fields on both sides of the metasurface, find the surface susceptibility values that satisfy (7) and (8).

\section{Absorbing Metasurface}

Let us now apply the above GSTCs to an absorbing metasurface.

\footnotetext{
${ }^{5}$ The normal susceptibility components have been ignored for simplicity. For the discussion on these normal susceptibilities see [33]. In addition, the GSTCs may be expressed in terms of surface impedances 34 or polarizabilities [25].
}

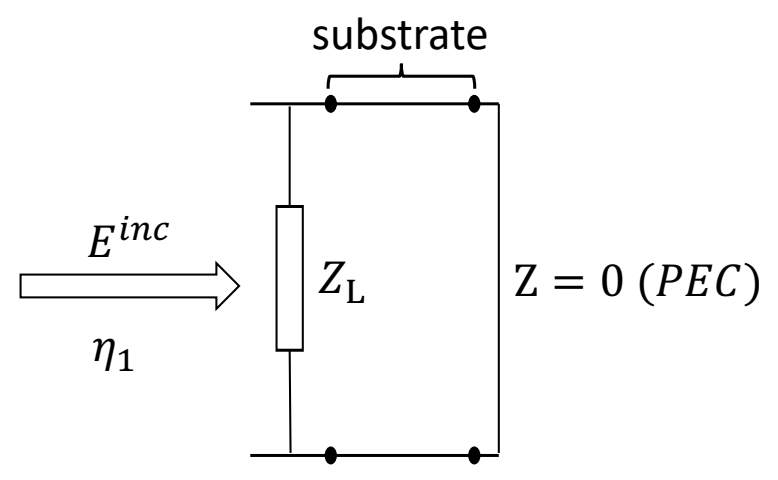

Fig. 3. The equivalent circuit form of a unit cell topology for a PECbacked absorbing metasurface. The complex load $Z_{L}$ may be implemented in different ways.

\section{A. Illumination from $x=0^{-}$side}

Considering Figure 2, and assuming that the metasurface needs to act as an absorbing metasurface, the desired tangential fields on the input side $\left(x=0^{-}\right)$of the metasurface must be equal to the incident field since the reflected field must ideally be zero. Therefore, noting that $E^{\mathrm{ref}}=H^{\mathrm{ref}}=0$, the tangential fields on the input side will be

$$
E_{z}^{-}=E_{z}^{\mathrm{inc}} \quad \text { and } \quad H_{y}^{-}=-H^{\mathrm{inc}} \cos \theta .
$$

Note that in Section [III we have assumed that the absorbing metasurface is metallic-backed. Assuming a perfect electric conductor (PEC) for the metal, the desired tangential fields on the output side $\left(x=0^{+}\right)$will be zero; i.e., $E_{z}^{+}=H_{y}^{+}=0$ (shielding). Since we now know the tangential fields on both side of the metasurface, we also know $\Delta E_{z}, \Delta H_{y}, E_{z, \text { av }}$ and $H_{y, \text { av }}$. Substituting these into (7) and (8) and also using the plane wave relation $E^{\text {inc }}=\eta_{1} \vec{H}^{\text {inc }}$, we arrive at

$$
\begin{gathered}
\cos \theta=\frac{1}{2} j \omega \varepsilon_{0} \eta_{1} \chi_{e e}^{z z}-\frac{1}{2} j \omega \sqrt{\mu_{0} \varepsilon_{0}} \cos \theta \chi_{e m}^{z y}, \\
-\eta_{1}=-\frac{1}{2} j \omega \mu_{0} \cos \theta \chi_{m m}^{y y}+\frac{1}{2} j \omega \sqrt{\mu_{0} \varepsilon_{0}} \eta_{1} \chi_{m e}^{y z} .
\end{gathered}
$$

\section{B. Illumination from $x=0^{+}$side}

Let us now assume that an incident field illuminates the metasurface from the other side, i.e., from $x=0^{+}$side. Since we have assumed that the metasurface is PEC-backed, this incident field will be totally reflected (i.e., shielding for the MWI system). Due to the shielding effect of the PEC, the fields on $x=0^{-}$will be zero, i.e., $E_{z}^{-}=H_{y}^{-}=0$. In addition, $E_{z}^{+}=0$, but $H_{y}^{+} \neq 0$. Then, 77 and 8 turn into

$$
\begin{gathered}
H_{y}^{+}=j \omega \sqrt{\mu_{0} \varepsilon_{0}} \chi_{e m}^{z y} \frac{H_{y}^{+}}{2}, \\
0=j \omega \mu_{0} \chi_{m m}^{y y} \frac{H_{y}^{+}}{2} .
\end{gathered}
$$




\section{Required Surface Susceptibilites}

From (12) and 13, we need to have $\chi_{m m}^{y y}=0$ and $\chi_{e m}^{z y}=$ $\frac{-2 j}{k_{0}}$. Noting that the reciprocity requires $\chi_{m e}^{y z}=-\chi_{e m}^{z y}[32]$, then we will have $\chi_{m e}^{y z}=\frac{2 j}{k_{0}}$. Finally, $\chi_{e e}^{z z}$ can be found from (10) as

$$
\chi_{e e}^{z z}=-\frac{4 j \cos \theta}{\omega \epsilon_{0} \eta_{1}} .
$$

Noting that $\cos \theta>0$ and assuming $\eta_{1} \in \mathbb{R}$, this set of surface susceptibilities indicates a lossy metasurface [35, Appendix $\mathrm{B}]$, which is expected for power dissipation 6

\section{Angular Dependency}

As can be seen from (14), the required surface susceptibility $\chi_{e e}^{z z}$ depends on the incidence angle $\theta$. Therefore, as it is well-known, the performance of the metasurface will have angular dependency. In MWI, since the object being imaged, represented by $O(\boldsymbol{r})$ in 2 , is unknown, we cannot predict the incidence angle of the wave impinging on the enclosure $\Gamma$. Based on the plane wave spectrum concept [36, Ch. 4], the fields emanating from the object being imaged can be thought as the summation of many plane waves each of which with a different incidence angle. Therefore, ideally, for MWI applications, it is desired to have an angular-independent absorbing metasurface which might be possible with an active metasurface [37]. However, since we use passive metasurfaces, the MWI reconstruction will be prone to this angular dependency. However, note that this angular-dependent absorption is still better than typical casings such as a metallic casing based on the level of the reflected signals.

\section{E. Unit Cell Topology}

It has been shown in [24], [22, Sect. 4], (and references therein) that this type of absorbing metasurfaces can be implemented using the unit cell topology whose equivalent circuit form is shown in Figure 3 As can be seen, this unit cell consists of a PEC-backed dielectric substrate with the load $Z_{L}$ printed on the substrate. Assuming a lossless dielectric substrate and normal incidence $(\theta=0)$, the load $Z_{L}$ will be

$$
Z_{L}=\left(\frac{1}{\eta_{1}}-\frac{1}{j Z_{\text {sub }} \tan \beta_{\text {sub }} t}\right)^{-1},
$$

where $Z_{\text {sub }}, \beta_{\text {sub }}$, and $t$ denotes the substrate's characteristic impedance, wavenumber, and thickness respectively. Note that $Z_{L}$ is a complex number. This load can be implemented in different ways, and therefore we may have different unit cell designs to achieve the same $Z_{L}$.

\section{Unit CELl Design}

As will be seen later on, we will consider three imaging examples. Each of these examples use a different absorbing metasurface. Herein, the unit cell for each of these examples are presented. (Later in Section VII these unit cells will be put together to form the enclosure $\Gamma$ of the MWI system.) Unit

\footnotetext{
${ }^{6}$ The polarizabilities of this type of absorbing metasurfaces have been derived in [22. Appendix A]. These polarizabilities can then be converted to the surface susceptibilities using [35. Appendix C].
}

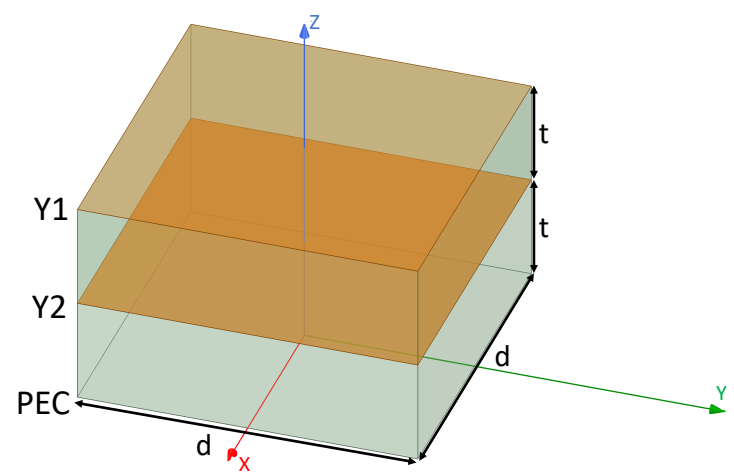

(a) Unit cell of Example I

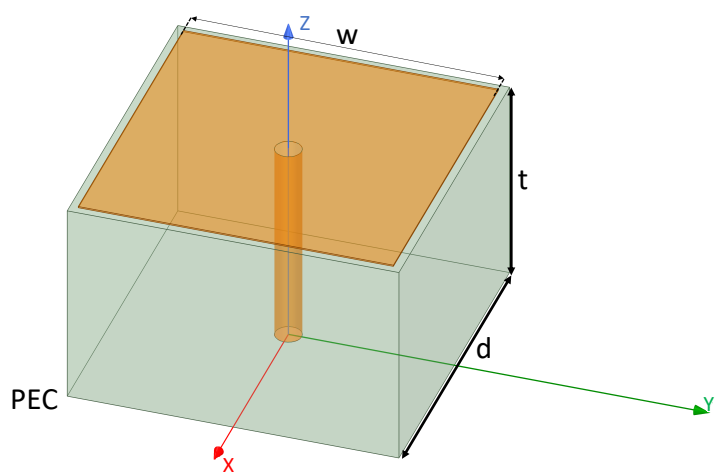

(b) Unit cell of Example II

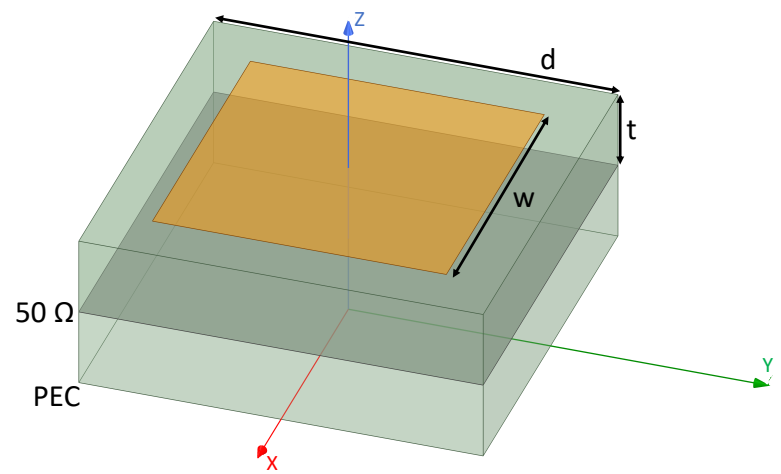

(c) Unit cell of Example III

Fig. 4. Three different unit cells for the three different imaging examples. (a) This unit cell consists of two Rogers RO3003 substrates with two admittance sheets denoted by Y1 and Y2 which are backed by a PEC plane. The size of the unit cell is denoted by $d$. The thickness of RO3003 substrate is denoted by $t$. (b) This unit cell, which is from [26], uses a PEC-backed lossy dielectric substrate of thickness $t=3 \mathrm{~mm}$, a $W \times W$ square copper patch $(W=4.75 \mathrm{~mm})$, and a cylindrical via of radius $0.2 \mathrm{~mm}$. The unit cell size is $d=5 \mathrm{~mm}$. (c) This unit cell consists of two RO3003 substrates. The top layer is a square PEC patch, and the middle layer consists of a resistive sheet.

cells are simulated using periodic boundary conditions and Floquet excitation ports in Ansys HFSS. The Floquet ports are placed sufficiently far from the unit cell to not include the effect of evanescent waves. Then, HFSS de-embedding is used to have the reference plane right on the unit cell structure. Due to the use of PEC-backed absorbing metasurfaces, only $S_{11}$ parameter will be considered to represent the reflection coefficient of the metasurface at the frequency of operation. 


\section{A. Unit Cell I}

As will be seen in Section VII-A in the first imaging example, the absorbing metasurface needs to work in a background medium with the relative complex permittivity of $75.6-j 4$ (water) at $1 \mathrm{GHz}$. This specific frequency and background permittivity are chosen based on the fact that in the first imaging example, water is assumed to be our coupling liquid. Note that the loss of the coupling liquid, which is represented by its imaginary part of the complex permittivity, is relatively small. Therefore, in the unit cell design process, $\epsilon_{b}$ has been assumed to be 75.6 for simplicity. (In the imaging algorithm, the loss of the coupling liquid is taken into account.) The structure of the unit cell employed for the first imaging example is shown in Figure 4(a) which consists of two admittance sheets $Y_{1}$ and $Y_{2}$. These two admittance sheets along with the PEC ground plane are on two identical Rogers RO3003 substrates with the thickness of $t=1.52 \mathrm{~mm}$ and the dielectric properties of $\epsilon_{r}=3$ and $\tan \delta=0.001$. In addition, the size of the unit cell, denoted by $d$ in Figure 4(a), is chosen to be $d=\lambda_{b} / 10=3.45 \mathrm{~mm}$, where $\lambda_{b}$ is the wavelength in the coupling liquid at the imaging frequency.

The design process requires determining the two admittance sheets $Y_{1}$ and $Y_{2}$. To this end, the transmission line model is used to determine the input admittance of the unit cell as

$$
Y_{1}+\left\{Z_{s} \frac{2 j \tan \left(\beta_{s} t\right) Z_{2}-\tan ^{2}\left(\beta_{s} t\right) Z_{s}}{j \tan \left(\beta_{s} t\right) Z_{s}+Z_{2}-Z_{2} \tan ^{2}\left(\beta_{s} t\right)}\right\}^{-1},
$$

where $Z_{s}$ is the characteristic impedance of the $\mathrm{RO} 3003$ substrate $7, \beta_{s}$ is the wavenumber within the substrate, and $Z_{2}=Y_{2}^{-1}$. This input admittance then needs to be matched with the admittance of the background medium. For the transmission line model, the impedance of the background medium 8 will be $\eta_{b}=120 \pi /(\sqrt{75.6} \cos \theta)$ [38]. Assuming $\theta=0$ (normal incidence), we then require 16 to be equal to $\eta_{b}^{-1}$ (impedance match) which results in

$$
Z_{1}=Y_{1}^{-1}=\eta_{b}
$$

and

$$
Z_{2}=Y_{2}^{-1}=\frac{-j Z_{S} \tan \left(\beta_{s} t\right)}{1-\tan ^{2}\left(\beta_{s} t\right)}
$$

As can be seen, $Z_{1}$ and $Z_{2}$ are purely resistive and capacitive respectively. In simulation, $Z_{1}$ and $Z_{2}$ are realized by HFSS impedance boundary conditions. (The values of $Z_{1}$ and $Z_{2}$ are $43.3278 \Omega$ and $-j 12.0559 \Omega$ ). As the incidence angle $\theta$ increases, the reflection coefficient $\left(\left|S_{11}\right|\right)$ increases as shown in Figure 5(a) 9 (Due to the use of impedance boundary conditions, as opposed to actual copper traces, this unit cell is less affected by the angular dependency.) Moreover, the frequency response of this unit cell has also been shown in Figure 5(b) demonstrating its narrow bandwidth.

\footnotetext{
${ }^{7}$ In the design process, $\tan \delta$ of the substrate, which is small, has been ignored for simplicity; however, in the HFSS simulations, it has been considered.

${ }^{8}$ As noted earlier, the imaginary part of the relative complex permittivity of the coupling liquid is ignored in the design process.

${ }^{9}$ Note that this is due to the fact that $\eta_{b}$ will be $120 \pi /(\sqrt{75.6} \cos \theta)$ instead of $120 \pi / \sqrt{75.6}$.
}

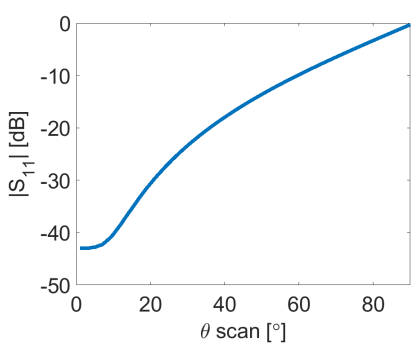

(a)

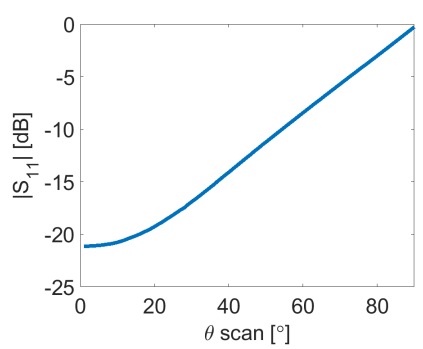

(c)

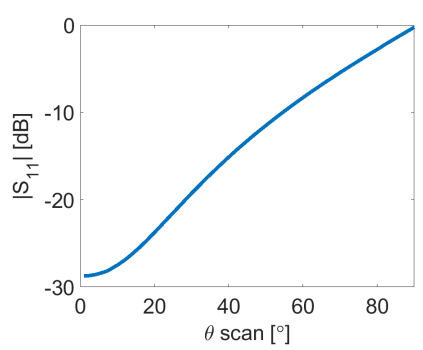

(e)

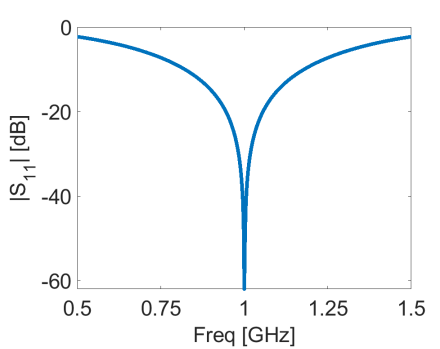

(b)

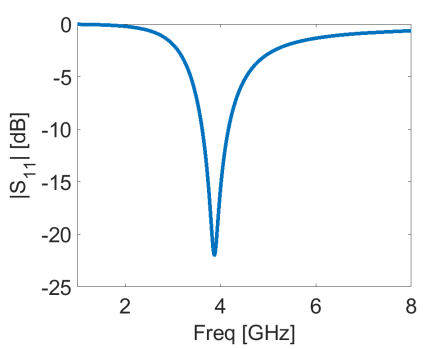

(d)

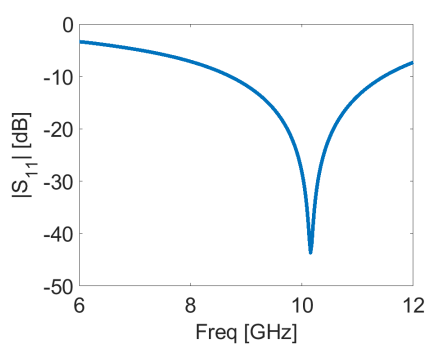

(f)
Fig. 5. Unit cell performance for the three examples: (Left Column) The reflection coefficient of the unit cell $\left|S_{11}\right|$ versus the incidence angle $\theta$. (Right Column) $\left|S_{11}\right|$ response versus the frequency of operation (assuming normal incidence). (a)-(b) Example I, (c)-(d) Example II, and (e)-(f) Example III.

\section{B. Unit Cell II}

As will be seen in Section VII-B, the background medium for the second imaging example is air. Even with the air background medium, an enclosure is required to support the system and/or mount the antennas; e.g., see [27] where 24 Vivaldi antennas operating in air have been mounted on a plexiglass enclosure. Herein, we use the mushroom unit cell proposed in [26] which operates at $3.844 \mathrm{GHz}$ and is shown in Figure 4 (b). The mushroom structure consists of (i) a PECbacked lossy substrate with a relative complex permittivity of $9-j 1.998$, (ii) a $4.75 \mathrm{~mm} \times 4.75 \mathrm{~mm}$ square $\frac{1}{2} \mathrm{oz}$. copper patch, and (iii) a cylindrical via with the radius of $0.2 \mathrm{~mm}$. In addition, we have $d=5 \mathrm{~mm}$ and $t=3 \mathrm{~mm}$. (The presence of the via makes the performance of the unit cell less angular dependent [26].) The reflection coefficient corresponding to different incidence angles can be found in Figure 5(c). In addition, the frequency response of the unit cell is shown in Figure $5(\mathrm{~d})$.

\section{Unit Cell III}

As will be seen in Section VII-C, in the third imaging example, the frequency of operation is set to $10 \mathrm{GHz}$, and the 


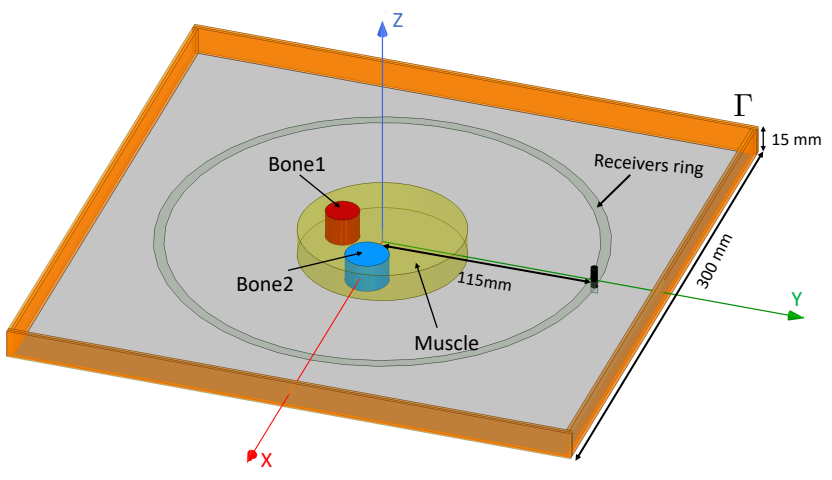

Fig. 6. Simulated MWI system for Target I. The enclosure of the system $\Gamma$ is an absorbing metasurface consists of the unit cell presented in Figure 4 a). The OI consists of a circular muscle tissue and two circular bones. The ring (measurement domain) over which the scattered data are measured and the transmitting line source located on the $y$ axis is also shown.

background medium is assumed to be air. For this example, we use a topology similar to Unit Cell I with Rogers RO3003 substrates with only one difference: swapping the capacitive and resistive layers. That is, the top layer is now capacitive with the impedance of $j X$ and the middle layer is now resistive with the impedance of $R$. (As will be seen in Section VIII. we use this model for our fabricated metasurface.) From the transmission line model, $R$ and $X$ for the normal incidence and a real-valued $\eta_{b}$ can be found as

$$
\begin{gathered}
R=\frac{\eta_{b} \sec ^{2}\left(\beta_{s} t\right) \pm \sqrt{\eta_{b}^{2} \sec ^{4}\left(\beta_{s} t\right)-16 Z_{s}^{2} \tan ^{2}\left(\beta_{s} t\right)}}{8}, \\
X=\frac{\eta_{b} Z_{s} \tan \left(\beta_{s} t\right)}{2 R-\eta_{b}} .
\end{gathered}
$$

Note that the resulting $R$ needs to be purely positive real $\left(R \in \mathbb{R}^{+}\right)$which requires $\eta_{b}^{2} \sec ^{4}\left(\beta_{s} t\right) \geq 16 Z_{s}^{2} \tan ^{2}\left(\beta_{s} t\right)$. For a given substrate, this might not hold at certain frequencies. In fact, at our frequency of operation, this condition is not met, and therefore we perform optimization using HFSS to find appropriate values. For the final unit cell design, we use two Rogers RO3003 substrates, each of which with the thickness of $t=1.52 \mathrm{~mm}$ as shown in Figure 4(c). On the top of the unit cell, there is a $5.73 \times 5.73 \mathrm{~mm}^{2}$ square PEC patch, which provides our capacitive value. (The impedance value of the layers can be tested individually, see [39].) Furthermore, at the middle layer, there is a $50 \Omega$ resistive sheet implemented using the HFSS impedance boundary condition ${ }^{10}$ At the bottom layer, there is a PEC layer which shields the metasurface. The unit cell response with respect to angle and frequency sweeps is presented in Figures 5(e) and (f) respectively. As can be seen in Figure 5 ff), due to the use of an approximated value for the middle layer impedance, the resonance frequency has a slight shift.

\footnotetext{
${ }^{10}$ Rogers provides resistive foil on their RO3003 laminate, which comes in 25,50 , and $100 \Omega$ per square (ps). Herein, the $50 \Omega$ resistive sheet is chosen due to its availability and the fact that it results in a reasonable absorption for the normal incidence for this unit cell. As will be seen in Section VIII we have used the $50 \Omega$ ps resistive foil for our fabricated metasurface.
}

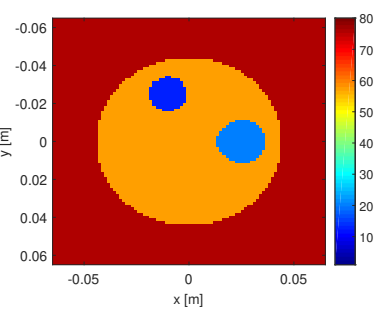

(a) $\operatorname{Re}\left(\epsilon_{r}\right)$, True profile

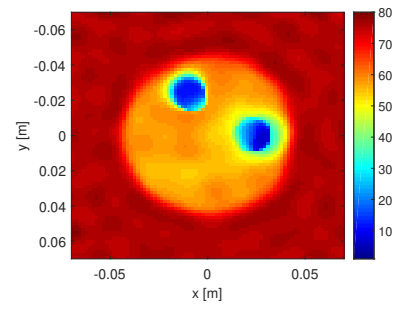

(c) $\operatorname{Re}\left(\epsilon_{r}\right)$, MR-GNI, PML

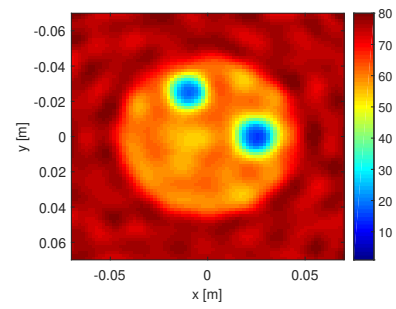

(e) $\operatorname{Re}\left(\epsilon_{r}\right)$, MR-GNI, Meta

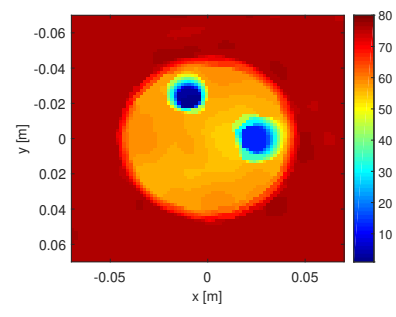

(g) $\operatorname{Re}\left(\epsilon_{r}\right)$, MR-CSI, PML

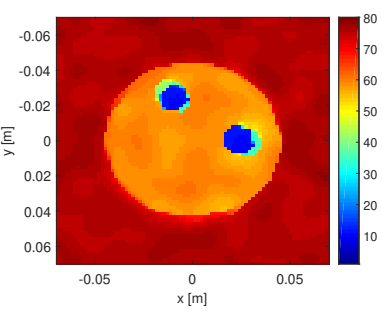

(i) $\operatorname{Re}\left(\epsilon_{r}\right)$, MR-CSI, Meta

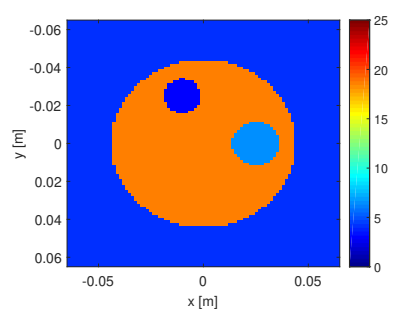

(b) $-\operatorname{Im}\left(\epsilon_{r}\right)$, True profile

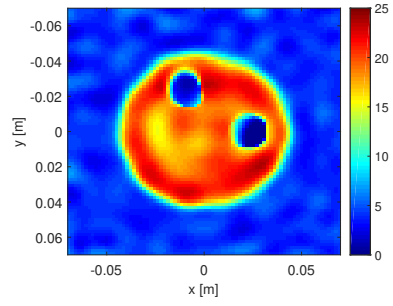

(d) $-\operatorname{Im}\left(\epsilon_{r}\right)$, MR-GNI, PML

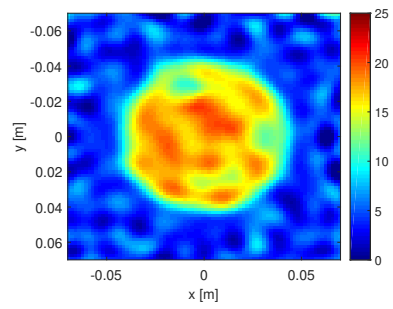

(f) $-\operatorname{Im}\left(\epsilon_{r}\right)$, MR-GNI, Meta

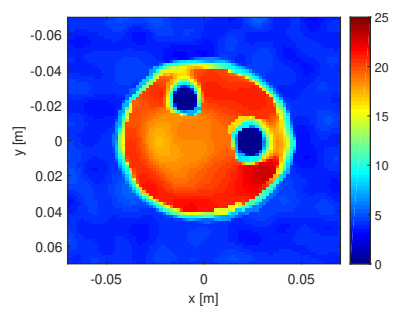

(h) $-\operatorname{Im}\left(\epsilon_{r}\right)$, MR-CSI, PML

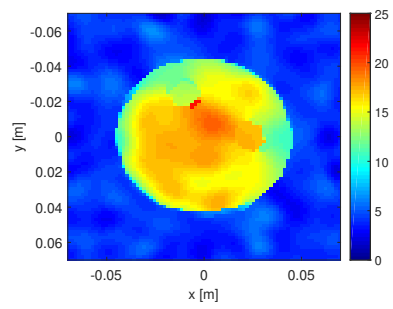

(j) $-\operatorname{Im}\left(\epsilon_{r}\right)$, MR-CSI, Meta
Fig. 7. (a)-(b) True relative complex permittivity profile for the synthetic human forearm (Target I). ('Re' and 'Im' denote the real-part and imaginary-part operators.) The MR-GNI results under (c)-(d) PML and (e)-(f) metasurface enclosure. The MR-CSI results under (g)-(h) PML and (i)-(j) metasurface enclosure.

\section{SyNThetic IMAGING ResUlts}

We consider three synthetic targets to demonstrate the performance of the absorbing metasurface enclosures. For all 


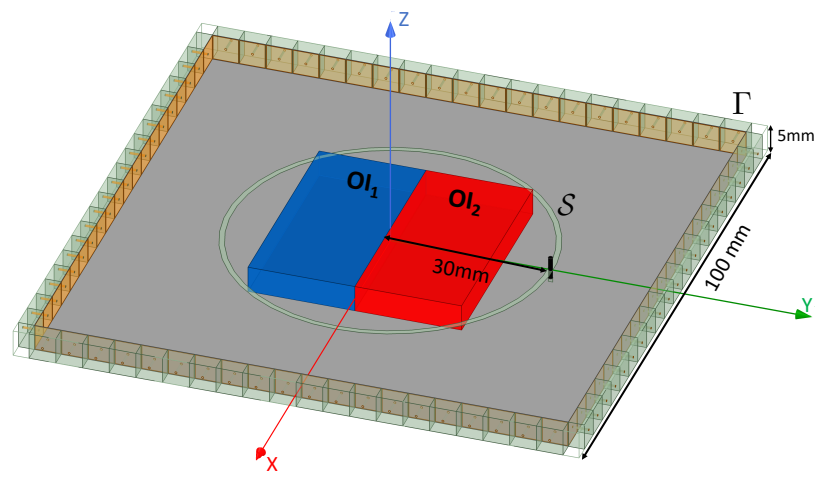

Fig. 8. Simulated MWI system for Target II. The enclosure of the system $\Gamma$ is an absorbing metasurface consists of the unit cell presented in Figure $4 \mathrm{~b}$ ). The target consists of two lossy dielectric rectangles. The measurement domain $\mathcal{S}$ is also shown.

these example, we utilize line sources to illuminate the targets. We then use Ansys HFSS to collect the scattered field data $E_{z}^{\text {scat }}$ on the measurement domain $\mathcal{S}$. These scattered data are then inverted by the imaging algorithm (e.g., the MR-GNI algorithm) to reconstruct the unknown dielectric profile $O(\boldsymbol{r})$ of the target. Since the imaging algorithms assume a 2D TM $z$ wave propagation, we place the simulation domain between two PEC planes to mimic an infinite length along the $z$ axis, thus, satisfying the assumption for $2 \mathrm{D} \mathrm{TM}_{z}$ propagation. To have references for comparison, we perform two other simulations for each target. For the first reference, we replace the absorbing metasruface enclosure with the HFSS perfectly matched layer (PML) boundary condition. The inversion of the data collected under the absorbing metasurface enclosure can then be compared with the inversion of the data collected under the PML boudary condition. For the second reference, we generate the scattered data set using a 2D MoM code that incorporates the green's function denoted in (3). The inversion of the data collected under the metasurface enclosure can also be compared with the inversion of the MoM free space data set.

In addition, note that the scale of the incident field in Ansys HFSS may be different than the scale of the incident field used in the imaging algorithm. To handle this, we utilize the incident field calibration [9], [29] for all the data generated by Ansys HFSS. That is, for a given transmitter, we compare the incident field utilized in the imaging algorithm with the incident field in Ansys HFSS over the measurement domain $\mathcal{S}$. From this comparison, for each transmitter, a calibration coefficient is formed to match the maximum of the modeled incident field with that of the HFSS incident field. This calibration coefficients will then be applied to the scattered data collected in Ansys HFSS when that particular transmitter is on. Once this process is done for all the transmitters, we now have our calibrated scattered data which will be given to the imaging algorithm to be inverted.

\section{A. Target I: Forearm}

This target, which has been depicted in Figure 6, consists of three cylinders. The large cylinder represents the muscle

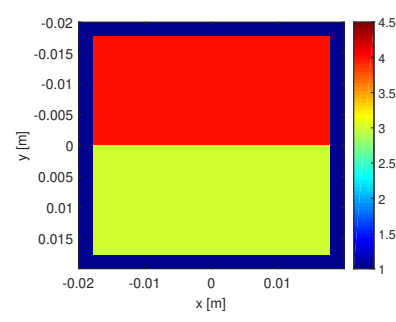

(a) $\operatorname{Re}\left(\epsilon_{r}\right)$, True profile

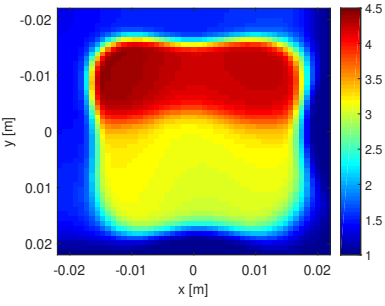

(c) $\operatorname{Re}\left(\epsilon_{r}\right)$, MR-GNI, Free space

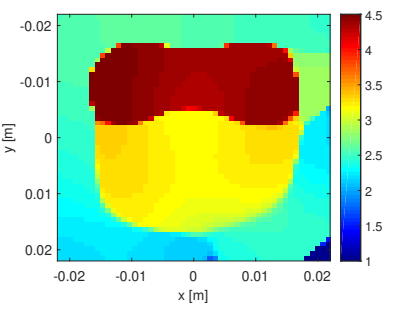

(e) $\operatorname{Re}\left(\epsilon_{r}\right)$, MR-GNI, Meta

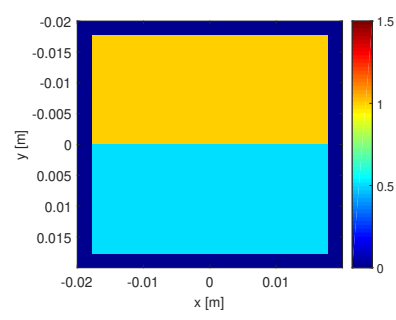

(b) $-\operatorname{Im}\left(\epsilon_{r}\right)$, True profile

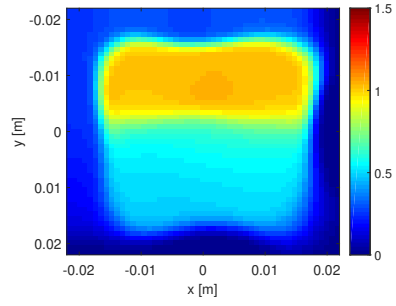

(d) $-\operatorname{Im}\left(\epsilon_{r}\right)$, MR-GNI, Free space

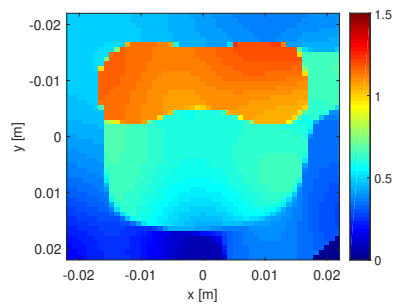

(f) $-\operatorname{Im}\left(\epsilon_{r}\right)$, MR-GNI, Meta
Fig. 9. (a)-(b) True relative complex permittivity profile of Target II. The MR-GNI reconstruction of the data set collected with the (c)-(d) free space condition and (e)-(f) metasurface enclosure.

tissue that encloses two bone tissue types. The bone tissues which have been denoted by Bone1 and Bone2 are depicted in red and blue in Figure 6 respectively. The relative complex permittivity of these two synthetic bones are $\epsilon_{r}=12.36-j 2.79$ and $\epsilon_{r}=20.58-j 6.54$ at $1 \mathrm{GHz}$ respectively. The muscle tissue, shown in yellow, has a relative complex permittivity $\epsilon_{r}=56.66-j 18.60$ at $1 \mathrm{GHz}$. The radii of the cylinders associated with Bone1, Bone2, and muscle are $9 \mathrm{~mm}, 11.5 \mathrm{~mm}$, and $44 \mathrm{~mm}$ respectively. This target has been immersed in a low-los ${ }^{11}$ coupling liquid having the relative complex permittivity of $\epsilon_{r}=75.6-j 4.0$ (water) at $1 \mathrm{GHz}$. The true real and imaginary parts of the relative complex permittivity of this target along with the background medium is shown in Figure 7(a)-(b) respectively. This target is interrogated by 24 transceiver ${ }^{12}$ (line sources) residing on receivers ring with the radius of $115 \mathrm{~mm}$ which is shown in Figure 6 at $1 \mathrm{GHz}$. Once one antenna transmits, the emanating $E_{z}$ field at the other 23 receiving locations are obtained. This

\footnotetext{
${ }^{11}$ In previous works [10], [12], the imaginary part of the coupling liquid's relative complex permittivity needed to be about $-j 15$ for successful imaging. Therefore, the use of a coupling liquid with an imaginary part of $-j 4$ is considered to be low-loss for this imaging problem.

${ }^{12}$ These are not co-resident; thus, challenges such as mutual coupling between the antenna elements are not considered. This is also true for the other examples.
} 


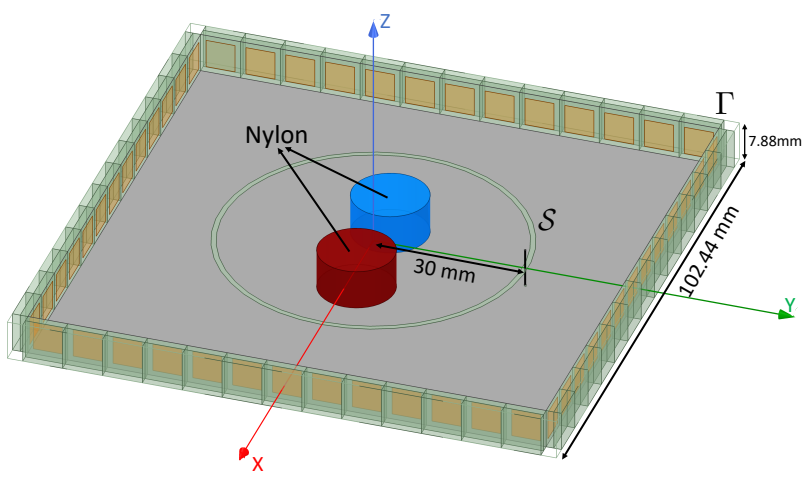

Fig. 10. Simulated MWI system for Target III. The enclosure of the system $\Gamma$ is an absorbing metasurface consists of the unit cell presented in Figure 4 (c) The OI consists of two lossless circular cylinders. The measurement domain $\mathcal{S}$ is also shown.

process results into $552(24 \times 23)$ collected total field data points. Subtracting the incident field $E_{z}^{\text {inc }}$ from the total field data results in 552 scattered data points that are to be processed using an inverse scattering algorithm to yield a quantitative relative complex permittivity image. The imaging domain is a $3.8 \times 3.8 \mathrm{~cm}^{2}$ domain discretized into $70 \times 70$ square cells. The MR-GNI inversion results under the PML and the metasurface enclosure have been shown in Figures 7 (e)-(h). The metasurface enclosure uses the unit cell described in Section VI-A and shown in Figure 4(a). As can be seen, the real-part reconstruction under the metasurface enclosure is close to that under the PML enclosure; however, the imaginary part reconstruction under the PML enclosure outperforms the imaginary part reconstruction under the metasurface enclosure. This is expected since the imaginary part reconstruction for biological targets is typically more difficult than the real part reconstruction [40], and often requires better signal-to-noise ratio. Finally, to make sure that the above results are not dependent on this specific imaging algorithm (MR-GNI), we have also inverted the data sets using the MR-CSI algorithm shown in Figures $7 \mathrm{~kg}$ )-(j). As can be seen, the results obtained with the MR-CSI algorithm are consistent with those obtained with the MR-GNI algorithm.

\section{B. Target 2: Two rectangular boxes}

This target, shown in Figure 8, consists of two attached lossy rectangular dielectric boxes. These two rectangular boxes, denoted by $\mathrm{OI}_{1}$ (in blue) and $\mathrm{OI}_{2}$ (in red), have the relative complex permittivity of $\epsilon_{r}=4.0-j 1.0$ and $\epsilon_{r}=3.0-j 0.5$ at the frequency of operation respectively. These two rectangular boxes have the same size of $3.490 \times 1.743 \mathrm{~cm}^{2}$. The background medium in this case study is air. We use 24 transceivers, located on a circle with the radius of $30 \mathrm{~mm}$, to irradiate this target at the frequency of $3.844 \mathrm{GHz}$. The metasurface enclosure uses the unit cell described in Section VI-B and shown in Figure 4(b). For this target, we compare the inversion performance under the metasurface enclosure with the case where there is no enclosure, i.e., the background medium (air) extends to infinity. For this 'no-enclosure' case, we use the MoM solver with (3) as its green's function to

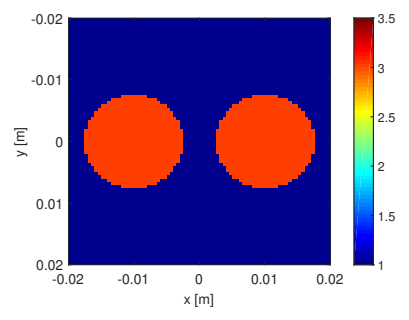

(a) $\operatorname{Re}\left(\epsilon_{r}\right)$, True profile

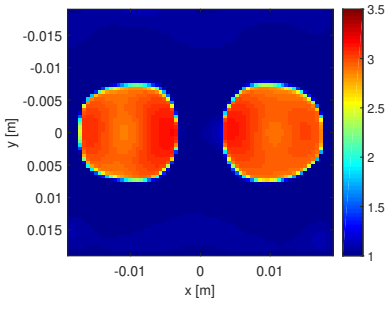

(c) $\operatorname{Re}\left(\epsilon_{r}\right)$, MR-GNI, PML

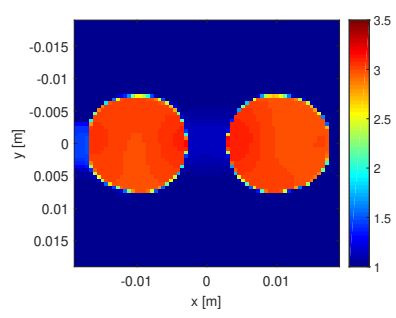

(b) $\operatorname{Re}\left(\epsilon_{r}\right)$, MR-GNI, Free space

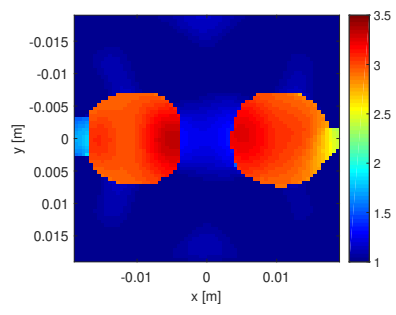

(d) $\operatorname{Re}\left(\epsilon_{r}\right)$, MR-GNI, Meta
Fig. 11. (a) True dielectric profile of Target III. (This target is lossless; thus, only the real part of the permittivity is shown.) The MR-GNI reconstruction of the data collected with the (b) free space condition, (c) PML boundary condition, and (d) metasurface enclosure.

generate the data, and we refer to it as 'free space' 13 The imaging domain is $4.4 \times 4.4 \mathrm{~cm}^{2}$ domain discretized into $51 \times 51$ square cells. The true real and imaginary parts of the relative complex permittivity of this target are shown in Figure 9(a)-(b) respectively. The MR-GNI results for the free space case and the metasurface enclosure have been shown in Figures 9(c)-(f). As can be seen, the inversion of the data collected under free space condition is more accurate; however, the inversion of the data collected with the metasurface enclosure still clearly shows the presence of the two dielectric rectangles but has image artefacts in the background medium. (The MR-CSI results are also consistent with the MR-GNI results, and thus are not shown for brevity.)

\section{Target 3: Two cylinders}

This target, shown in Figure 10, consists of two lossless dielectric cylinders each of which has a radius of $7.5 \mathrm{~mm}$ and the relative complex permittivity of $\epsilon_{r}=3.0$ (lossless) at the frequency of operation. These two cylinders have been separated from each other by $5 \mathrm{~mm}$. The background medium in this case study is air. We use 24 transceivers, evenly distributed on a circle with the radius of $30 \mathrm{~mm}$, to irradiate the target at the frequency of $10 \mathrm{GHz}$. The true target is shown in Figure 11 a). (Since the object is lossless, the imaginary part of the relative complex permittivity is zero and is thus not shown for brevity.) The imaging domain is $3.8 \times 3.8 \mathrm{~cm}^{2}$ domain discretized into $70 \times 70$ square cells.

Three different scattering data sets are considered: (i) free space data set, (ii) data under the PML, and (iii) data under the metasurface enclosure with the unit cells described in

\footnotetext{
${ }^{13}$ The MoM data set is generated on a different grid than the one used in the inversion algorithm to avoid the inverse crime.
} 


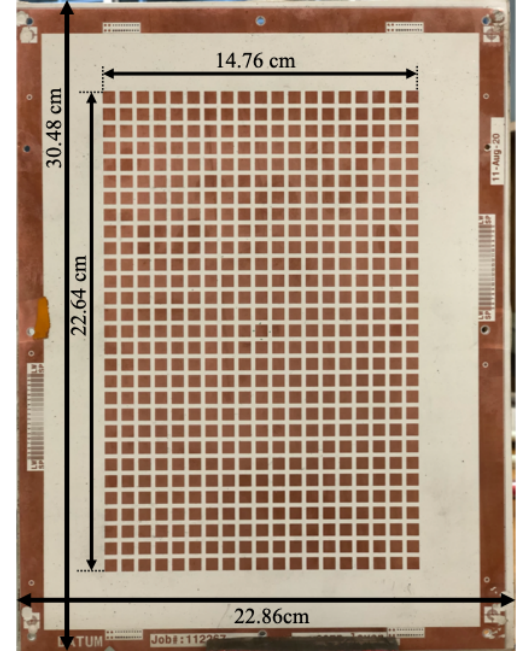

(a)

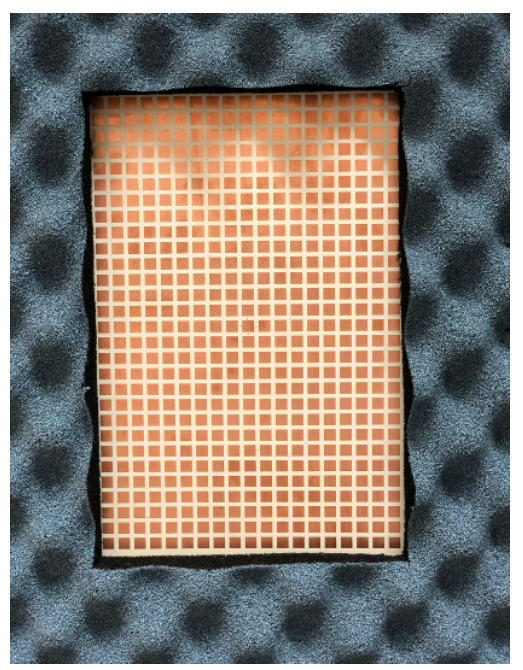

(b)

Fig. 12. (a) The fabricated metasurface which uses two Rogers RO3003 substrates, one of which including a $50 \Omega$ per square resistive foil bonded by an RO2929 bondply and (b) the same fabricated metasurface placed in an absorber panel to cover its margins, thus, only exposing its $29 \times 19$ square patch array.

Section VI-C and shown in Figure 4 (c). The MR-GNI results for these three data sets are shown in Figure 11(b)-(d). As can be seen, the inversion of the free space and PML data sets are very accurate. In addition, the inversion of the data collected under the metasurface enclosure is also accurate but has some small artefacts.

\section{EXPERIMENTAL RESULTS}

Herein, we first present the fabricated metasurface and then describe how we have measured its reflectivity for the normal incidence.

\section{A. Fabrication}

An absorbing metasurface based on the configuration of Unit Cell III, described in Section VI-C, was fabricated, which is shown in Figure 12 a). This metasurface consists of two

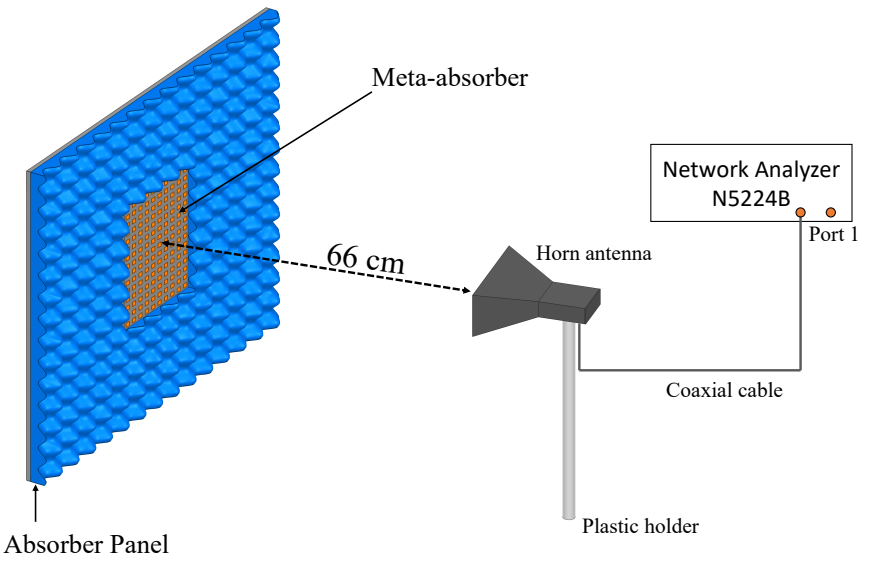

Fig. 13. The measurement setup. A horn antenna is used to irradiate the metasurface. A vector network analyzer, using its time-domain option (time gating), records the reflected signal from the metasurface for the normal incidence.

Rogers RO3003 substrates (thickness of $t=1.52 \mathrm{~mm}$ and the dielectric properties of $\epsilon_{r}=3$ and $\tan \delta=0.001$ ) which are bonded together by a Rogers RO2929 bondply. This bondply has a relative permittivity of 2.94 and a dissipation factor of 0.003 at $10 \mathrm{GHz}$. For our fabrication, we have used an RO2929 with the thickness of 3 mil which is about $0.08 \mathrm{~mm}$. The length and width of the substrates are cut to 12 in $(30.48 \mathrm{~cm})$ and 9 in $(22.86 \mathrm{~cm})$, respectively. On the top substrate, there exist 551 $(29 \times 19) \frac{1}{2}$ oz square copper patches. Similar to Section VI-C the size of the patches is chosen to be $5.73 \times 5.73 \mathrm{~mm}^{2}$ with a separation of $2.15 \mathrm{~mm}$ between two neighboring patches. In addition, for ease of handling, some empty space from the outline of the square patches to the board outline has been left.

For the bottom substrate, we have used Rogers RO3003 that comes with a 50 ohms per square (ps) resistive foil. One side of this particular substrate consists of an OhmegaPly RCM, which stands for resistor-conductor material [41]. The other side of this substrate has a typical copper layer. The OhmegaPly layer consists of Nickel Phosphorous (NiP) metal alloy with the thickness of $18 \mu \mathrm{m}$ electrodeposited onto a copper layer [41]. This metal alloy serves as our resistive sheet. For our fabrication, we have removed the copper layer in the vicinity of the resistive foil. However, the other copper layer remains untouched as the absorbing metasurface is a metallic backed structure.

For the top substrate, we have used a typical RO3003 substrate (i.e., without any resistive foil). For our fabrication, the copper layers on both side of the top substrate have been removed. This top substrate is then bonded (using 3 mil Rogers RO2929 bondply) to the bottom substrate so as to form the final metasurface shown in Figure 12 a). Thus, in summary, our metasurface consists of the followings

$$
\begin{aligned}
\text { Metasurface }= & \text { Copper layer (ground) }+ \text { RO3003 dielectric } \\
& +50 \Omega \text { ps Resistive foil }+ \text { RO2929 bondply } \\
& + \text { RO3003 dielectric }+ \text { Copper patches. }
\end{aligned}
$$

Finally, to experimentally evaluate the performance of the 


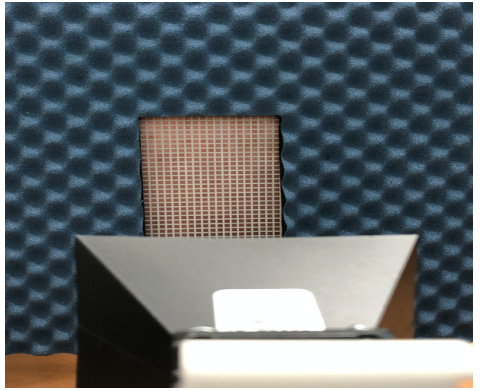

(a)

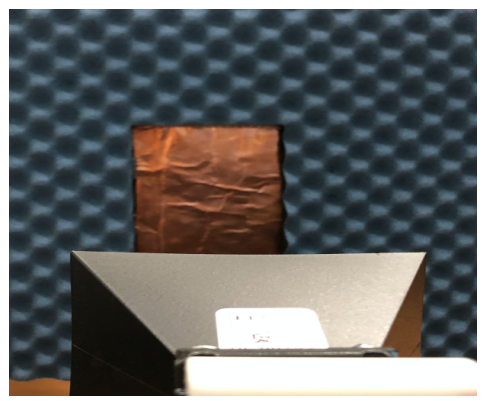

(b)

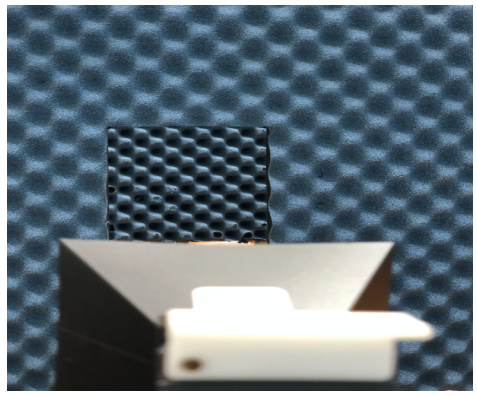

(c)

Fig. 14. Horn antenna irradiating (a) the metasurface and (b) the copper tape placed in front of the metasurface. In the last case, i.e., the "free-space" case shown in (c), we have removed the metasurface from the absorber panel, thus having an absorbing panel with a rectangular opening. To reduce the reflections due to this opening, an extra absorbing panel has been placed behind the rectangular opening in (c).

metasurface, we place it within an absorber panel as shown in Figure 12 (b). Based on this placement, we now merely see the array of $29 \times 19$ square patches.

\section{B. Measurements}

The schematic of the measurement setup is presented in Figure 13 where a horn antenna is used to irradiate the metasurface. (Visually, we have tried to have the metasurface and the horn aperture in parallel.) The distance between the horn aperture to the metasurface is $66 \mathrm{~cm}$ The horn is fed through Port 1 of a vector network analyzer (VNA) which in our case is a Keysight PNA Network Analyzer N5224B. Figure 14(a) shows the irradiation of the metasurface by

\footnotetext{
${ }^{14}$ At the frequency of $10 \mathrm{GHz}$, this distance corresponds to about $22 \lambda$ where $\lambda$ denotes the wavelength of operation. In addition, the $2 D^{2} / \lambda$ farfield criterion where $D$ denotes the maximum size of the horn antenna is about $58 \mathrm{~cm}$. Therefore, based on the above two criteria, we can assume that the metasurface is in the far-field zone of the horn antenna.
}

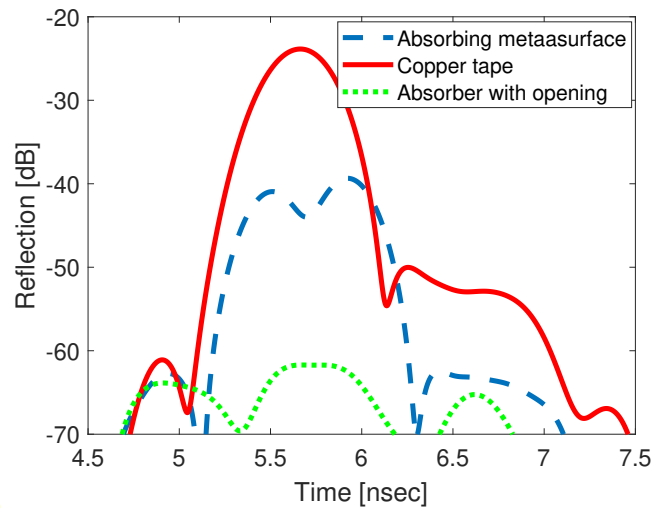

(a)

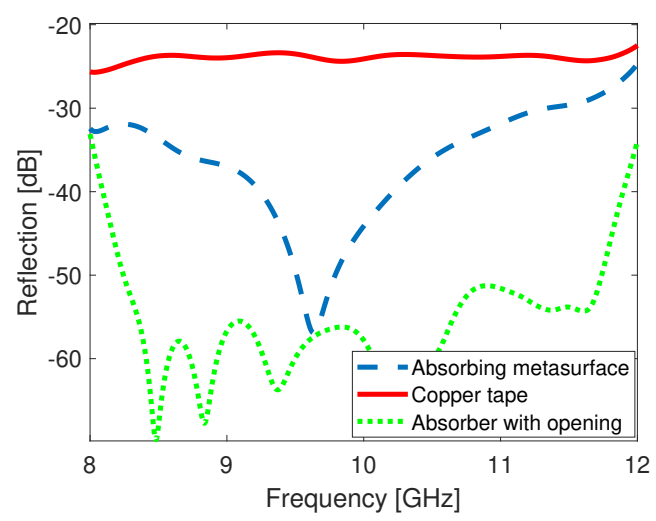

(b)

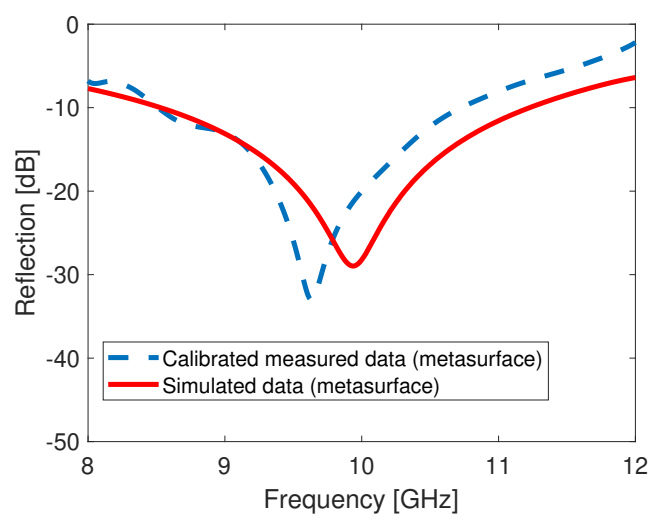

(c)

Fig. 15. (a) The reflection as a function of time for the absorbing metasurface, copper tape, and free space cases within the time gating interval. (b) The reflection as a function of frequency for these three cases when the time gating is on. (c) The calibrated measured reflection from the absorbing metasurface compared to its corresponding simulated data in Ansys HFSS.

the horn antenna assuming the normal incidence. One-port calibration (from $8 \mathrm{GHz}$ to $12 \mathrm{GHz}$ with 6401 points, $0 \mathrm{dBm}$ power level, and an IF bandwidth of $10 \mathrm{KHz}$ ) was performed to have the reference plane at the feeding point of the horn antenna. The time-domain feature of the VNA was used to find the reflectivity of the metasurface, similar to [42]. Turning on the time-domain option, we noticed a reflected signal greater than $-60 \mathrm{~dB}$ at around $5.19 \mathrm{nsec}$. Given that the distance 


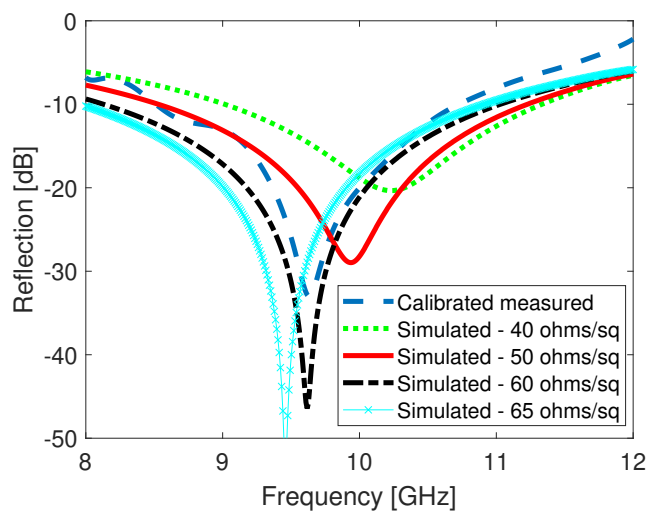

Fig. 16. The calibrated measured reflection from the absorbing metasurface compared to its corresponding simulated data in Ansys HFSS for varying sheet resistance value from $40 \mathrm{ohms}$ per square to $65 \mathrm{ohms}$ per square.

from the feeding point (VNA calibrated reference plane) to the horn aperture is about $13 \mathrm{~cm}$, the total round-trip distance from the reference plane to the metasurface will then be $2 \times(66+13)$ or $158 \mathrm{~cm}$ which corresponds to an arrival time of about 5.27 nsec. Therefore, we were convinced that this is the reflection emanating from the metasurface. The time gating $^{15}$ was then applied from $4.5 \mathrm{nsec}$ to $7.5 \mathrm{nsec}$. That portion of this time interval which corresponds to reflections greater than or equal $-70 \mathrm{~dB}$ is shown in Figure 15 (a) in blue (dashed curve). To be able to compare this reflection with a reference metallic reflecting surface, we place copper tape in front of the absorbing metasurface as shown in Figure 14(b). Using the same time gating, the reflection emanating from this metallic surface is shown in Figure 15 (a) in red (solid curve) ${ }^{16}$ In addition, to have a "free space" reference, we remove the metasurface from the absorber panel, thus, now having an absorber panel with a rectangular opening. To minimize possible reflections from the wall and nearby objects going toward the horn antenna through this rectangular opening, we then place an extra absorber panel behind the rectangular opening as shown in Figure 14(c). We refer to this case as the "free space" case. Using the same time gating as above, the reflection from this configuration is shown in Figure 15.a) in green (dotted curve). By comparing the three curves in Figure $15(\mathrm{a})$, we can easily see that the reflection emanating from the metasurface (as a function of time) is smaller than that of the metallic surface but greater than the reflection in the free space case.

Having time gating on, we then go back to the frequency domain and look at the reflection coefficient as a function of frequency for both the absorbing metasurface (blue) and copper tape (red) as well as the free space (green) cases as shown in Figure 15. b). As can be seen, around the frequency of $9.63 \mathrm{GHz}$, the absorbing metasurface has about $33 \mathrm{~dB}$ less

\footnotetext{
${ }^{15}$ For the time gating, we have used the following VNA settings: Gate Type and Gate Shape are set to Band Pass and Normal respectively.

${ }^{16} \mathrm{To}$ prevent the fabricated metasurface from possible damages, we just place the copper tape in front of the metasurface without peeling off its paper-backing. Therefore, the copper tape does not sit completely flush on the surface of the metasurface.
}

reflection as compared to the reflection from the copper tape case. In addition, at the same frequency, the reflection from the metasurface is very similar to the reflection associated with the free space case. These two comparisons confirm that this is the operational frequency of the fabricated metasurface.

Finally, to compare the experimental performance of this metasurface with its simulated data, we need to calibrate our measured data. To this end, knowing that the reflection coefficient from a perfect electric conductor must be $0 \mathrm{~dB}$, we apply additive calibration numbers (in $\mathrm{dB}$ ) to each reflection value of the copper tape case (red) shown in Figure 15.b) so as to convert it to a flat line at $0 \mathrm{~dB}$ as a function of frequency. We then apply the same additive calibration numbers to the curve associated with the reflections from the metasurface as a function of frequency. This will result in the blue curve (dashed) shown in Figure 15.c) which is referred to as the calibrated reflection and varies from about $-2 \mathrm{~dB}$ to $-33 \mathrm{~dB}$. The simulated reflection from this metasurface using Ansys HFSS has also been shown in red (solid) in Figure 15 . c ${ }^{17}$ As can be seen, the simulated data has a similar trend to the measured data with the main difference being the shift in its frequency of operation. We speculate that one reason behind this shift in the operational frequency could be due to the change that the resistive value may undergo in the fabrication process. To investigate this possibility, we have performed simulations with four different sheet resistance values. As can be seen in Figure 16 the calibrated measured reflection, shown in dashed (dark) blue, is quite close to the simulation result if the sheet resistance is considered to be $60 \mathrm{ohms}$ per square (dashed-dot black) as opposed to the original 50 ohms per square (solid red).

\section{CONCLUSION}

In MWI system design, it is often desired (i) to reduce the reflections happening at the enclosure of the imaging system, and (ii) to shield the imaging chamber from external interference. To this end, one option is to use a metallic enclosure in conjunction with a lossy coupling liquid such as salt water. The undesired reflected waves from the enclosure will then need to go through a lossy background medium to arrive back at the receivers, thus being weakened. The main advantage of this approach is the simplicity of its implementation which has also shown successful experimental imaging results. On the other hand, its main disadvantage is its negative effect on the desired signal level, and consequently worsening the overall signal-to-noise ratio of the measured data. This paper proposes an alternative approach which does not require a lossy background medium. To this end, PECbacked absorbing metasurfaces were utilized as the enclosure of simulated MWI systems. The PEC-backed structure shields the imaging chamber from external interference. In addition, the absorbing metasurface reduces the level of the reflected waves from the enclosure. This approach comes with its own disadvantage: narrow bandwidth. In addition, the absorption

\footnotetext{
${ }^{17}$ Note that as opposed to the simulation result presented in red in Figure 15 (c), the simulation result shown in Figure 5 f) does not take into account the bondply, and also assumes PEC for the copper patches.
} 
of these metasurfaces is a function of incidence angles, which is a challenge for complex scattering events in the imaging chamber. To alleviate this in this paper, the transmitting antenna was placed not too close to the metasurface enclosure.

Herein, three different unit cell models have been considered. Except Unit Cell II, the other two models use Ansys HFSS' impedance boundary conditions for the implementation of the resistive layer (and, the capacitive layer in Unit Cell I). It is expected that these simple models produce better results than what can be obtained using practical implementations of the resistive and capacitive components. Therefore, the imaging results obtained under these simulated metasurfaces need to be treated as the upper bounds of the achievable image accuracy. Nevertheless, this paper shows that the use of absorbing metasurfaces in microwave imaging can be useful for shielding the imaging system from external interference, and at the same time, reducing the undesired reflections from the system casing. In particular, for those microwave imaging applications where the background medium must be air and yet the system needs to be shielded by an enclosure, such PEC-backed absorbing metasurfaces can be employed as the system enclosure.

Finally, an absorbing metasurface using two bonded Rogers substrates was fabricated and measured for the normal incidence. The preliminary results are promising. However, we still need to experimentally investigate the performance of this absorbing metasurface in an imaging chamber, which is a much more complex environment, to see how close this enclosure can mimic a free space condition while still shielding the imaging chamber from external interference.

\section{ACKNOWLEDGMENT}

The authors would like to thank the Canadian Microelectronics Corporation for the provision of Ansys HFSS, and Rogers Corporation for providing the substrate materials used in fabrication. The financial support of the Natural Sciences and Engineering Research Council (NSERC) of Canada, University of Manitoba's UMGF and GETS Program, as well as the Canada Research Chair (CRC) Program is also acknowledged. The authors would also like to thank Mr. Ehsan Zeynali for his help with the measurement work.

\section{REFERENCES}

[1] P. M. Meaney, M. W. Fanning, T. Raynolds, C. J. Fox., Q. Fang, C. A. Kogel, S. P. Poplack, and K. D. Paulsen, "Initial clinical experience with microwave breast imaging in women with normal mammography," Acad Radiol., March 2007.

[2] N. K. Nikolova, Introduction to Microwave Imaging. United Kingdom: Cambridge University Press, 2017.

[3] M. Pastorino, Microwave Imaging. New Jersey: John Wiley \& Sons, 2010.

[4] E. C. Fear, S. C. Hagness, P. M. Meaney, M. Okoniewski, and M. A. Stuchly, "Enhancing breast tumor detection with near-field imaging," IEEE Microwave Mag., vol. 3, no. 1, pp. 48-56, Mar 2002.

[5] R. Palmeri, M. T. Bevacqua, L. Crocco, T. Isernia, and L. D. Donato, "Microwave imaging via distorted iterated virtual experiments," IEEE Transactions on Antennas and Propagation, vol. 65, no. 2, pp. 829838, Feb 2017.

[6] A. Abubakar, T. M. Habashy, G. Pan, and M. K. Li, "Application of the multiplicative regularized Gauss Newton algorithm for threedimensional microwave imaging," IEEE Transactions on Antennas and Propagation, vol. 60, no. 5, pp. 2431-2441, May 2012.
[7] P. Mojabi and J. LoVetri, "Microwave biomedical imaging using the multiplicative regularized Gauss-Newton inversion," IEEE Antennas Wireless Propag. Lett., vol. 8, pp. 645-648, 2009.

[8] A. Abubakar, P. M. van den Berg, and J. J. Mallorqui, "Imaging of biomedical data using a multiplicative regularized contrast source inversion method," IEEE Trans. Microwave Theory Tech., vol. 50, no. 7 , pp. 1761-1777, July 2002.

[9] M. Ostadrahimi, P. Mojabi, C. Gilmore, A. Zakaria, S. Noghanian, S. Pistorius, and J. LoVetri, "Analysis of incident field modeling and incident/scattered field calibration techniques in microwave tomography," IEEE Antennas Wireless Propag. Lett., vol. 10, pp. 900-903, 2011.

[10] M. Ostadrahimi, P. Mojabi, A. Zakaria, J. LoVetri, and L. Shafai, "Enhancement of Gauss-Newton inversion method for biological tissue imaging," IEEE Transactions on Microwave Theory and Techniques, vol. 61 , no. 9, pp. 3424-3434, Sept 2013.

[11] P. M. Meaney, C. J. Fox, S. D. Geimer, and K. D. Paulsen, "Electrical characterization of glycerin:water mixtures: Implications for use as a coupling medium in microwave tomography," IEEE Trans. Microw. Theory Tech., vol. 65, no. 5, pp. 1471-1478, May 2017.

[12] C. Gilmore, A. Zakaria, J. LoVetri, and S. Pistorius, "A study of matching fluid loss in a biomedical microwave tomography system," Medical Physics, vol. 40, pp. 1-14, 2013.

[13] T. Rubæk, P. M. Meaney, P. Meincke, and K. D. Paulsen, "Nonlinear microwave imaging for breast-cancer screening using Gauss-Newton's method and the CGLS inversion algorithm," IEEE Trans. Antennas Propag., vol. 55, no. 8, pp. 2320-2331, Aug 2007.

[14] P. M. van den Berg and J. T. Fokkema, "Removal of undesired wavefields related to the casing of a microwave scanner," IEEE Trans. Microw. Theory Tech., vol. 51, no. 1, pp. 187-192, Jan 2003.

[15] M. Asefi, A. Baran, and J. LoVetri, "An experimental phantom study for air-based quasi-resonant microwave breast imaging," IEEE Transactions on Microwave Theory and Techniques, vol. 67, no. 9, pp. 3946-3954, 2019.

[16] C. Pfeiffer and A. Grbic, "Metamaterial Huygens' surfaces: Tailoring wave fronts with reflectionless sheets," Phys. Rev. Lett., vol. 110, p. 197401, May 2013.

[17] C. L. Holloway, E. F. Kuester, J. A. Gordon, J. O'Hara, J. Booth, and D. R. Smith, "An overview of the theory and applications of metasurfaces: The two-dimensional equivalents of metamaterials," IEEE Antennas and Propagation Magazine, vol. 54, no. 2, pp. 10-35, 2012.

[18] M. Selvanayagam and G. Eleftheriades, "Discontinuous electromagnetic fields using orthogonal electric and magnetic currents for wavefront manipulation," Optics Express, pp. 14 409-14 429, 2013.

[19] T. Brown, Y. Vahabzadeh, C. Caloz, and P. Mojabi, "Electromagnetic inversion with local power conservation for metasurface design," IEEE Antennas and Wireless Propagation Letters, 2020.

[20] G. Lavigne, K. Achouri, V. S. Asadchy, S. A. Tretyakov, and C. Caloz, "Susceptibility derivation and experimental demonstration of refracting metasurfaces without spurious diffraction," IEEE Transactions on Antennas and Propagation, vol. 66, no. 3, pp. 1321-1330, 2018

[21] M. Del Mastro, M. Ettorre, and A. Grbic, "Dual-band, orthogonallypolarized lp-to-cp converter for satcom applications," IEEE Transactions on Antennas and Propagation, pp. 1-1, 2020.

[22] Y. Ra'di, C. R. Simovski, and S. A. Tretyakov, "Thin perfect absorbers for electromagnetic waves: Theory, design, and realizations," Phys. Rev. Applied, vol. 3, p. 037001, Mar 2015.

[23] G. Xu, S. V. Hum, and G. V. Eleftheriades, "Augmented Huygens' metasurfaces employing baffles for precise control of wave transformations," IEEE Transactions on Antennas and Propagation, vol. 67, no. 11, pp. 6935-6946, June 2019.

[24] N. Engheta, "Thin absorbing screens using metamaterial surfaces," in IEEE APS International Symposium, vol. 2, June 2002, pp. 392-395.

[25] Y. Ra'di, V. S. Asadchy, and S. A. Tretyakov, "Total absorption of electromagnetic waves in ultimately thin layers," IEEE Transactions on Antennas and Propagation, vol. 61, no. 9, pp. 4606-4614, Sep. 2013.

[26] O. Luukkonen, F. Costa, C. R. Simovski, A. Monorchio, and S. A. Tretyakov, "A thin electromagnetic absorber for wide incidence angles and both polarizations," IEEE Transactions on Antennas and Propagation, vol. 57, no. 10, pp. 3119-3125, Oct 2009.

[27] C. Gilmore, P. Mojabi, A. Zakaria, M. Ostadrahimi, C. Kaye, S. Noghanian, L. Shafai, S. Pistorius, and J. LoVetri, "A wideband microwave tomography system with a novel frequency selection procedure," IEEE Transactions on Biomedical Engineering, vol. 57, no. 4, pp. 894-904, April 2010.

[28] Z. Liu, N. Bayat, and P. Mojabi, "On microwave imaging with absorbing metasurface enclosure," in 2020 IEEE International Symposium on 
Antennas and Propagation and North American URSI Radio Science Meeting, 2020, pp. 1-2.

[29] A. Abubakar, P. M. van den Berg, and T. M. Habashy, "Application of the multiplicative regularized contrast source inversion method on TMand TE-polarized experimental Fresnel data," Inverse Probl., vol. 21, pp. S5-S13, 2005.

[30] M. M. Idemen, Discontinuities in the electromagnetic field. John Wiley $\&$ Sons (IEEE Press series on electromagnetic wave theory; 40), 2011.

[31] E. F. Kuester, M. A. Mohamed, M. Piket-May, and C. L. Holloway, "Averaged transition conditions for electromagnetic fields at a metafilm," IEEE Transactions on Antennas and Propagation, vol. 51, no. 10, pp. 2641-2651, 2003

[32] K. Achouri, M. A. Salem, and C. Caloz, "General metasurface synthesis based on susceptibility tensors," IEEE Trans. Antennas Propag, vol. 63 , no. 7, pp. 2977-2991, July 2015.

[33] K. Achouri and O. J. F. Martin, "Angular scattering properties of metasurfaces," IEEE Transactions on Antennas and Propagation, vol. 68, no. 1, pp. 432-442, 2020.

[34] A. Epstein and G. V. Eleftheriades, "Huygens' metasurfaces via the equivalence principle: design and applications," J. Opt. Soc. Am. B, vol. 33, no. 2, pp. A31-A50, Feb 2016.

[35] K. Achouri, "Synthesis and applications of electromagnetic metasurfaces," Ph.D. dissertation, École Polytechnique de Montr “eal, Montreal, Canada, 2017.

[36] S. Gregson, J. McCormick, and C. Parini, Principles of Planar NearField Antenna Measurements. United Kingdom: The Institution of Engineering and Technology (IET), 2007.

[37] G. Lavigne and C. Caloz, "Angle-independent nongyrotropic metasurfaces," arXiv:1903.11956, 2019.

[38] J. P. S. Wong, A. Epstein, and G. V. Eleftheriades, "Reflectionless wideangle refracting metasurfaces," IEEE Antennas and Wireless Propagation Letters, vol. 15, pp. 1293-1296, 2016.

[39] G. Xu, S. V. Hum, and G. V. Eleftheriades, "Augmented huygens' metasurfaces employing baffles for precise control of wave transformations," IEEE Transactions on Antennas and Propagation, vol. 67, no. 11, pp. 6935-6946, 2019.

[40] P. Mojabi and J. LoVetri, "A prescaled multiplicative regularized GaussNewton inversion," IEEE Transactions on Antennas and Propagation, vol. 59, no. 8, pp. 2954-2963, Aug 2011.

[41] Ohmega Technologies, Inc., "Ohmegaply® product selection guide," https://ohmega.com/wp-content/uploads/Ohmega-Brochure-01012016. pdf

[42] M. Yoo and S. Lim, "Polarization-independent and ultrawideband metamaterial absorber using a hexagonal artificial impedance surface and a resistor-capacitor layer," IEEE transactions on antennas and propagation, vol. 62, no. 5, pp. 2652-2658, 2014. 